\title{
Atomic and Molecular Databases, VAMDC
}

\author{
M. L. Dubernet ${ }^{1}$, C. M. Zwölf ${ }^{1}$, N. Moreau ${ }^{1,}$, Y. A. Ba ${ }^{1}$ and \\ VAMDC Consortium ${ }^{2}$ \\ ${ }^{1}$ LERMA, Observatoire de Paris, PSL Research University, CNRS, UMR8112, 5 Place \\ Janssen, 92195 Meudon, France \\ email: marie-lise.dubernet@obspm.fr \\ ${ }^{2}$ http://www.vamdc.eu
}

\begin{abstract}
The VAMDC Consortium is a worldwide consortium which federates Atomic and Molecular databases through an e-science infrastructure and a political organisation. About $90 \%$ of the inter-connected databases handle data that are used for the interpretation of spectra and for the modeling of media of many fields of astrophysics. This paper presents how the VAMDC Consortium is organised in order to publish atomic and molecular data for astrophysics.
\end{abstract}

Keywords. databases, atomic data, molecular data

\section{Introduction}

The VAMDC Consortium originates from two European funded projects: the VAMDC (http://www.vamdc-project.vamdc.eu/)(Dubernet et al. 2010) and the SUP@VAMDC (http://www.sup-vamdc.vamdc.org/)(Zwölf et al.2014) projects. The main scientific outcomes of those two projects are: 1) an e-science infrastructure that interconnects about thirty databases (http://www.vamdc.eu/activities/research); 2) a political and technical organisation: "the VAMDC Consortium" that was launched on the 1rst November 2014 through the signature of a Memorandum of Understanding (MoU) between 15 partners. This structure ensures the organisation and the sustainability of the VAMDC activities. This MoU is complemented by an "Internal Regulations" Document describing in detail the implementation of the MoU and a Roadmap that provides the general strategy of the VAMDC Consortium $\dagger$. The VAMDC Consortium activities cover 4 domains: research which is the most developed domain, education and industry that are currently in their early development stage, and outreach activities.

\section{The Research Services}

The Research Services are organised towards offering a common entry point to all databases thanks to the VAMDC portal (http://portal.vamdc.eu), towards offering the possibility to include new data and new databases within the VAMDC e-infrastructure, towards providing software librairies and modules that can be included into customers software, towards providing standalone users oriented software that retrieve and handle atomic and molecular data. The VAMDC e-infrastructure has evolved over the years through successive releases (Rixon et al.(2011), Doronin et al.(2012), Dubernet et al.(2015)) in order to arrive to the current version of standards and software (release version v12.07).

The current VAMDC e-infrastructure includes databases related to atomic and molecular spectroscopy and to heavy particle collisional processes, and is appropriate to the type of currently accessible data. Any producer of data can join the VAMDC infrastructure

$\dagger$ http://www .vamdc.eu - see section "About us"/How to join us 
through different means: 1) they may include their data in existing atomic and molecular databases that are partners of VAMDC; 2) they may create a new database hosted by a partner of VAMDC; 3) they may create a new node in the VAMDC e-infrastructure. In cases 1 and 2, the data producers can contact the databases managers directly, while the general support system (support@vamdc.eu) should be contacted in the third case. Furthermore VAMDC aims to provide atomic and molecular data providers and compilers with a large dissemination platform for their work through its communication tools (website, forum) and through its citation policy. Currently all products related to VAMDC, portal and tools, explicitly warn that the VAMDC users should cite both the original papers where the data have been published and the relevant databases.

The librairies, software modules and software can be found on the VAMDC website (http://www.vamdc.eu/software). The integration of those librairies are documented, supported via tutorials and illustrated in scientific use cases (Dubernet et al. 2014). Users might want to create new librairies and software, and we provide support for those activities (support@vamdc.eu). In addition the VAMDC Consortium can provide on-demand additional innovative tools for easily handling and processing results.

\section{Conclusion}

The VAMDC Consortium continuously welcomes new members and is opened to welcome new type of data. Two main motivations would be considered in order to extend the scope of VAMDC Consortium: 1) a new community of data provider is interested to benefit from our experience and from part of our software, 2) one of our user community needs different types of data to be combined with the set of data already available in the VAMDC e-infrastructure. The inclusion of new types of data would certainly impact some of the "VAMDC Consortium" members, therefore the "VAMDC Consortium" members supporting such changes should make a case showing that this community is strategic for reasons such as increase of visibility, new customers, new stakeholders leading to consolidation of sustainability.

\section{Acknowledgements}

The VAMDC Support has been provided through the VAMDC and the SUP@VAMDC projects funded under the "Combination of Collaborative Projects and Coordination and Support Actions" Funding Scheme of The Seventh Framework Program. Call topic: INFRA-2008-1.2.2 and INFRA-2012 Scientific Data Infrastructure. Grant Agreement numbers: 239108 and 313284.

\section{References}

Doronin, M., Dubernet, M. L., Walton, N., et al. 2012, in Astr. Soc. of the Pacific Conf. Series, Vol. 461, Astronomical Data Analysis Software and Systems XXI, 331

Dubernet, M. L., Aboudarham, J., Ba, Y. A., et al. 2014, in SF2A-2014: Proceedings of the Annual meeting of the French Society of Astronomy and Astrophysics, 17-23

Dubernet, M. L., Boudon, V., Culhane, J. L., et al. 2010, J. Quant. Spect. Rad. Trans., 111, 2151

Dubernet, M. L., Rixon, G., \& Doronin, M., VAMDC Collaboration 2015, Highlights of Astronomy, 16, 685

Rixon, G., Dubernet, M. L., Piskunov, N., et al. 2011, in AIP Conf. Series, Vol. 1344, ed. A. Bernotas, R. Karazija, \& Z. Rudzikas, 107-115

Zwölf, C., Dubernet, M.-L., Ba, Y., \& Moreau, N., VAMDC Consortium 2014, in IEEE, ISTAfrica Conference Proceedings 